\title{
Terrain Characterization and Classification with a Mobile Robot
}

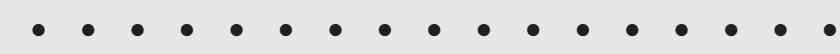

\author{
Lauro Ojeda and Johann Borenstein \\ Department of Mechanical Engineering \\ The University of Michigan \\ Ann Arbor, Michigan 48109 \\ e-mail:lojeda@umich.edu, johannb@umich.edu
}

\section{Gary Witus}

Turing Associates, Inc.

1392 Honey Run Drive

Ann Arbor, Michigan 48103

e-mail: witusg@umich.edu

\author{
Robert Karlsen \\ U.S. Army TARDEC \\ 6501 East 11 Mile Road \\ AMSRD-TAR/R (MS 263) \\ Warren, Michigan 48397 \\ e-mail:karlsenr@tacom.army.mil
}

Received 29 November 2005; accepted 8 January 2006

\begin{abstract}
This paper introduces novel methods for terrain classification and characterization with a mobile robot. In the context of this paper, terrain classification aims at associating terrains with one of a few predefined, commonly known categories, such as gravel, sand, or asphalt. Terrain characterization, on the other hand, aims at determining key parameters of the terrain that affect its ability to support vehicular traffic. Such properties are collectively called "trafficability." The proposed terrain classification and characterization system comprises a skid-steer mobile robot, as well as some common and some uncommon but optional onboard sensors. Using these components, our system can characterize and classify terrain in real time and during the robot's actual mission. The paper presents experimental results for both the terrain classification and characterization methods. The methods proposed in this paper can likely also be implemented on tracked robots, although we did not test this option in our work. ๑ 2006 Wiley Periodicals, Inc.
\end{abstract}

Parts of Section 4 of this paper were presented at the SPIE Defense and Security Conference, Unmanned Ground Vehicle Technology VII, Orlando, Florida, March 28-April 1, 2005.

Journal of Field Robotics 23(2), 103-122 (2006) (C) 2006 Wiley Periodicals, Inc. 


\section{INTRODUCTION}

Most research on off-road mobile robot sensing focuses on obstacle negotiation, path planning, and position estimation. These issues have conventionally been the foremost factors limiting the performance and speeds of mobile robots. Very little attention has been paid to date to the issue of terrain classification, which aims at associating terrain with well-defined categories, such as gravel, sand, or dirt.

A related but different type of terrain analysis is terrain characterization, that is, determining characteristics of the terrain that affect the driving performance and safety of small mobile robots traversing the terrain. Yet, trafficability is of great importance if mobile robots are to reach speeds that human-driven vehicles can reach on rugged terrain. For example, it is obvious that the maximal allowable speed for a turn is lower when driving over sand or wet grass than when driving on packed dirt or asphalt. In order to emphasize that the characterization methods discussed in this paper relate to the trafficability of the terrain, we use the term "terrain trafficability characterization" throughout this paper.

The main difference between characterization and classification is that characterization tells us how the terrain affects driving behavior (e.g., slippery and soft) without attempting to identify the terrain. Terrain classification, on the other hand, does not necessarily tell us how the terrain affects driving behavior, but it tells us what type of terrain it is. An example for the significance of this distinction is this: In order for a mobile robot to drive safely but at the highest possible speed over an asphalt road, it is very important to know whether the asphalt is wet or dry. It is less important to know that the terrain is made of asphalt, as long as the robot knows what cornering forces or break distance the terrain will support. In contrast, a remote human operator may want to know if the robot is still driving over grass or if it has reached a gravel-covered parking lot adjacent to a targeted building. In this example, terrain classification can help pinpoint the location of the robot on, say, an aerial map that the operator is using.

In the remainder of this section, we review relevant earlier research about terrain classification and characterization.

\subsection{Terrain Classification}

In the context of this paper, "terrain classification" is the act of identifying the type of the terrain being traversed, from among a list of candidate terrains.

Early work on terrain classification-not specifically for robotics applications-focused on analyzing the texture of video images and synthetic aperture radar images. A survey of work performed with these two types of sensors can be found in Weszka, Dyer \& Rosenfeld (1976) and Belhadj, Saad, El Assad, Saillard \& Barba (1994), respectively. Another popular sensor modality for terrain classification is video; because video cameras are small, lightweight, emit no detectable energy, and they are inexpensive. Examples for work on wheeled mobile robots can be found in Talukder et al. (2002), and for legged robots in Larson, Voyles \& Demir (2004). With the arrival of three-dimensional range sensors, researchers have also used range information for terrain classification purposes (Vandapel, Huber, Kapuria \& Hebert, 2004).

Our proposed terrain classification system uses typically available on-board sensors, such as gyros, accelerometers, encoders, as well as motor current and voltage sensors. In addition, the paper describes a number of less commonly used sensors and their effectiveness with regard to terrain classification. These sensors are downward-facing ultrasonic and infrared sensors, as well as microphones. Signals from each sensor modality are fed into trained neural networks (NNs), one NN for each sensor modality. The output of each $\mathrm{NN}$ is a number between zero and one that indicates the likelihood of the present terrain being one of five previously defined and trained terrains. Somewhat related work has been proposed by Sadhukhan (2004) and Brooks, Iagnemma \& Dubowsky (2005). However their work was limited to the use of accelerometers.

\subsection{Terrain Characterization}

Terrain characterization has been the subject of several studies, although these earlier studies were typically aimed at larger vehicles and the characterization process was manual. Perhaps the best known and widely cited works are those of Bekker (1956, 1960, 1969) and Wong (2001). From the point of view of terramechanics, soil can be characterized by determining the terrain parameters. Many approaches to terrain characterization require offline analysis 
and/or dedicated equipment (Nohse et al., 1991; Shmulevich, Ronai \& Wolf, 1996). Terrain characterization without dedicated equipment was proposed for the Sojourner rover and its 1997 Mars mission (Matijevic et al., 1997). Based on this method, Sojourner used one of its wheels to characterize terrain. A real-time approach based on the measurement of wheel sinkage in soft soil using a video camera was proposed in Iagnemma, Kang, Shibly \& Dubowsky (2004).

In this paper, we propose a fully self-contained terrain characterization method for skid-steer mobile robots. With "self-contained" we mean that our system does not require any special-purpose instruments to be attached to the robot. Rather, the proposed method monitors typical onboard sensors, such as gyros and motor current sensors. The unique advantage of this approach is that it can be applied during real time and during an actual robot mission. In numerous runs, we collected data on five different terrains: Gravel, sand, asphalt, grass, and dirt. Sensor data were collected while the robot performed carefully prescribed maneuvers. We then analyzed the data with our proposed method, which yielded a curve that is characteristic for a particular terrain.

\section{THE EXPERIMENTAL PLATFORM}

The mobile robot used in all experiments was a Pioneer 2-AT (P2AT), shown in Figure 1. The robot was equipped with a large number of sensor modalities. We categorize these sensors as follows:

1. Inertial Sensors (see Figure 2): This system, developed originally at the University of Michigan, is also know and referred to as the FLEXnav Proprioceptive Position Estimation (PPE) system (Ojeda, Reina \& Borenstein, 2004). The FLEXnav PPE system used in this project comprises:

- Two medium-quality Coriolis gyros for the $x$ and $y$ axis;

- One high-quality fiber-optic gyro for the $z$ axis; and

- Three single-axis accelerometers.

2. Motor Sensors: Two current and two voltage sensors for measuring torque and momentary power consumption for the left-hand and right-hand side motors. While the P2AT

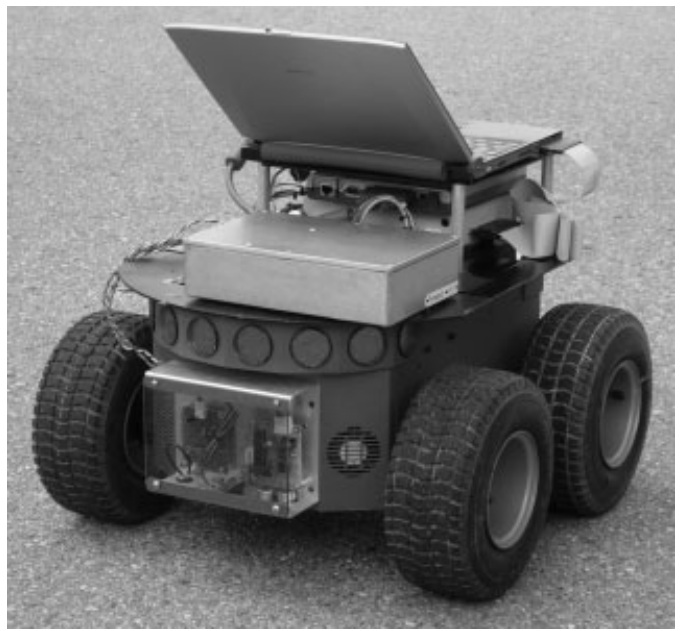

Figure 1. This Pioneer 2-AT (P2AT) was used in all experiments.

has a pair of drive motors on each side, each pair is mechanically coupled and acts effectively as one motor.

3. Range Sensors (see Figure 3):

- One ultrasonic range sensor, which uses a $23 \mathrm{kHz}$ ultrasonic transmitter and one receiver; and

- One infrared range sensor.

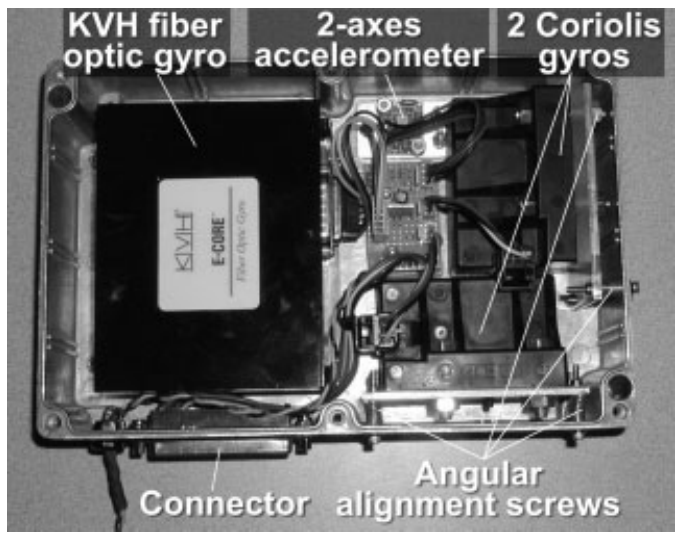

Figure 2. The Inertial Navigation Unit (IMU) built for this project comprises a KVH fiber optic gyro for the $z$ axis and two Coriolis gyros for the $x$ and $y$ axes, as well as a two-axes accelerometer. 


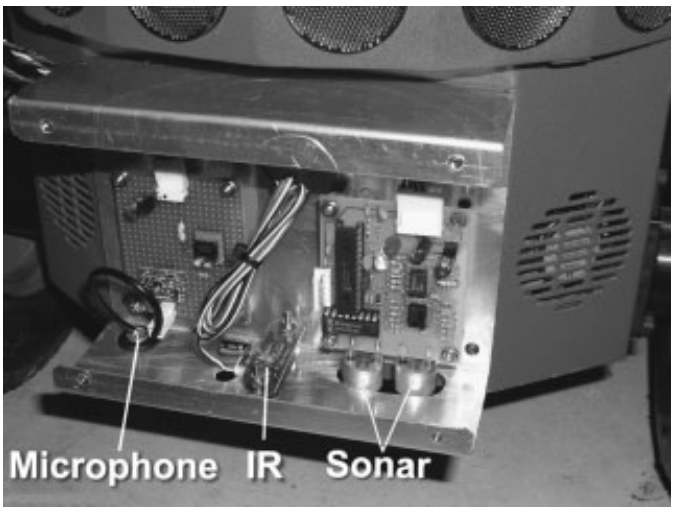

Figure 3. Three sensor modalities were mounted in front of the robot, pointing downward: A microphone, an infrared range sensor, and an ultrasonic range sensor.

\section{Other Sensors:}

- One microphone (see Figure 3); and

- Two wheel encoders.

A laptop computer provided the computing power, and data acquisition was performed using a 16-input 16-bit PCMCIA data acquisition card. Figure 4 shows a hardware diagram of the complete system, comprising the P2AT platform, computers, and sensors. Software developed specifically for this project includes a library of motion primitives that perform a number of predefined motions. These motions are described in more detail in the following sections.

\section{TERRAIN CLASSIFICATION}

In our study, we attempted to identify which one of five different candidate terrains the robot traveled on: Gravel, grass, sand, pavement, or dirt. Our hypothesis was that each terrain type has a unique signature when "viewed" by a certain sensor modality. The output signals from each one of the available sensor modalities were sampled and stored for subsequent offline analysis in MATLAB. The analysis consisted of data preprocessing, as needed for each sensor modality, followed by classification, by means of a NN. The $\mathrm{NN}$ is explained in greater detail in the following section. It is perfectly feasibly to do the preprocessing and NN analysis in real time; although in our work here, we used MATLAB functions for convenience.

\section{Pioneer 2-AT Computer Topography}

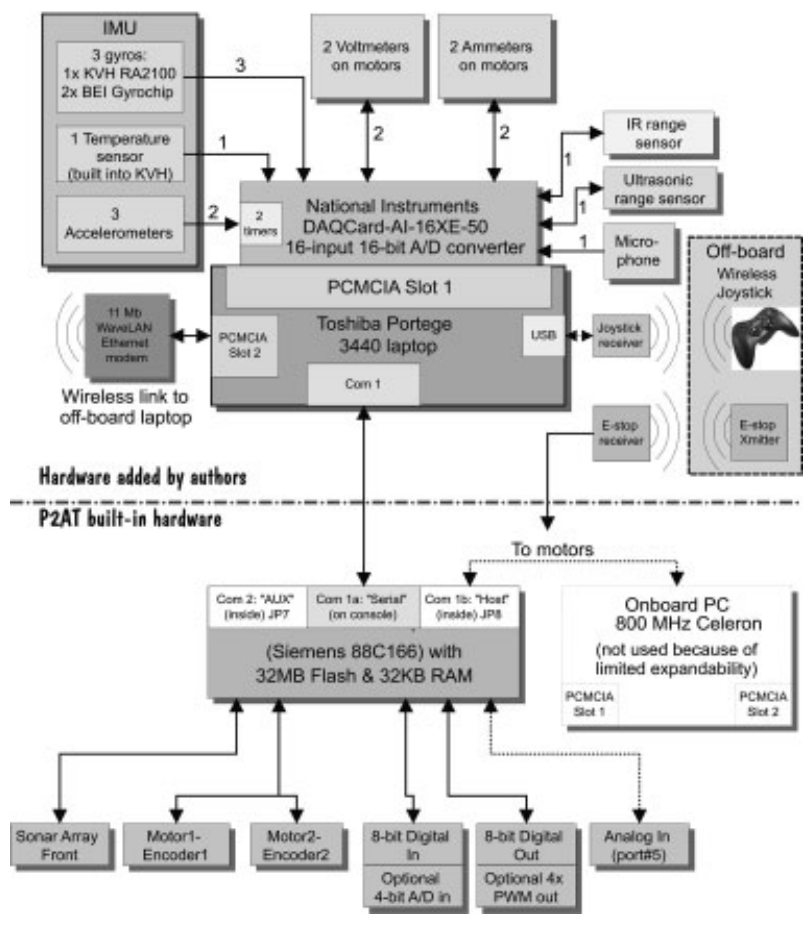

Figure 4. Pioneer 2-AT hardware components.

\subsection{Neural Networks for Terrain Classification}

In recent years, applications using NNs have proven to be successful in many areas. Most of them are in the field of pattern recognition. Multilayer feedforward networks are universal approximators (Hornik, Stinchcombe \& White, 1989), and can approximate any function with any desired degree of accuracy, provided that an adequate number of hidden processing elements are available. Therefore, we can expect a properly dimensioned and trained NN to perform as an efficient classifier. NNs present several advantages that make them desirable for pattern classification applications. NNs can represent linear and nonlinear models and learn those relations directly from the training data. In addition, they can generalize this knowledge to new situations. NNs are not dependent on statistical distributions or spectral responses and can be made tolerant to noise variations.

We performed all described terrain classification experiments using a feed-forward $\mathrm{NN}$ with five out- 


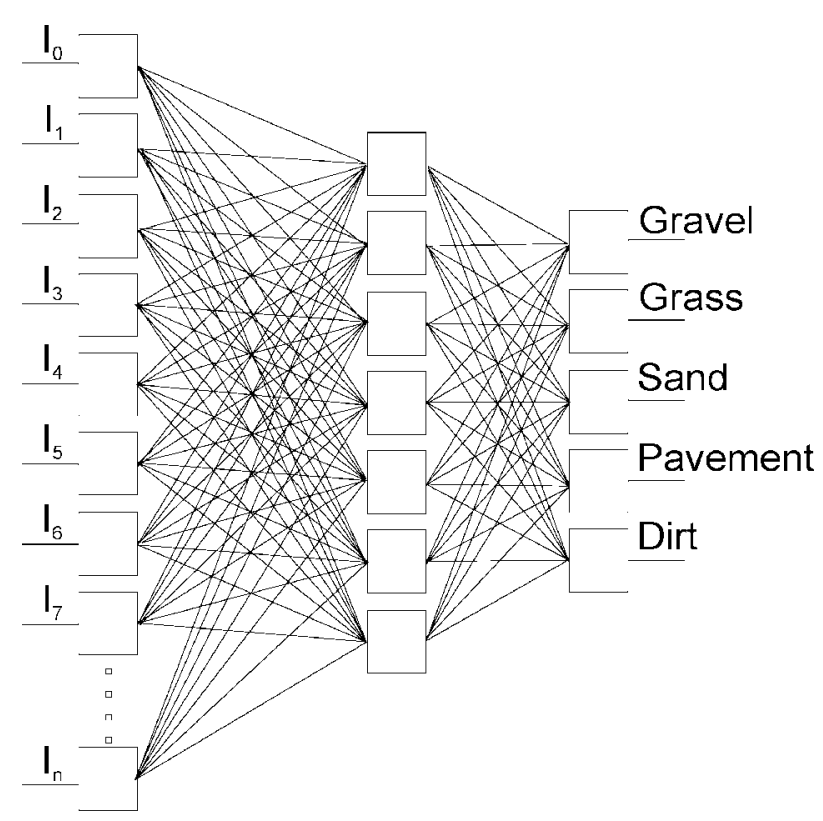

Figure 5. Structure of the neural net used for terrain classification.

puts, as shown in Figure 5. Each output can vary from zero to one, in proportion to the likelihood that a given signal presented in the input of the NN belongs to one of the five subject terrains: Gravel, grass, sand, pavement, or dirt.

All classification experiments were performed based on just one single sensor modality at a time. That is, one NN per signal of interest was created, trained, and tested. Although data from several different sensor modalities can be fed into one single NN, we refrained from doing so. This was in order to avoid increasing the number of neurons in the $\mathrm{NN}$, which would have increased the size and training time considerably. Alternatively, and more likely to be successful, one can combine the outputs of multiple sensor-specific NNs to improve the final overall performance. This is particularly true when the sensors characteristics are complementary. However, implementation and testing of such multi-NN systems was beyond the scope of this project.

The number of $\mathrm{NN}$ inputs, $n$, can vary depending on the sensor modality being tested and the preprocessing applied to the signal. In all cases, we first used the discrete Fourier transform (DFT) to decompose the signal into its frequency components, and the result of the DFT was then used as the input for the NN. The DFT is the discrete or finite implementation of the Fourier transform, which can be used to represent a signal as a function of sinusoidal basis functions. We computed the DFT of the input signal at fixed intervals of 128 samples. This is essentially a periodogram, which in turn is an approximation of the signal power spectrum or power spectrum density. For a detailed explanation of the DFT and periodogram see Papoulis (1991). The sampling frequency of the signals was $200 \mathrm{~Hz}$, and the output of each DFT was made up of 64 components representing the frequency content in the range from 0 to $100 \mathrm{~Hz}$. For most of the signals, we used $n=32$ inputs. This number of inputs corresponds to frequencies from 0 to $50 \mathrm{~Hz}$. In the few cases where sensor data were used in the time domain, we set $n=25$ inputs. Regardless of the number of inputs, all tested NNs had one intermediate layer. In our analysis, we also tried NNs with two intermediate layers, but found that this did not improve the classification performance.

For each terrain, we performed two separate experiments; each one on two different locations that were at least $3 \mathrm{~m}$ apart from each other. In all cases, we used one data set for training the $\mathrm{NN}$ and the other one for testing it. For each NN, the number of process elements per layer was determined by carefully tuning the size of the NN until it provided the best classification performance. However, regardless of the quality of the tuning, the NN accuracy is greatly reduced when signals corresponding to different terrains have similar signatures, as will be seen later. In that case, the NN does not classify the terrains correctly.

There is a tradeoff in selecting the appropriate number of process elements. If too many are selected, the NN learns to classify the training data set correctly, but it performs poorly with the test data set. On the other hand, if the number of neurons is too small, the NN is not able to learn all the patterns. Therefore, as we designed each NN, we started with a large $\mathrm{NN}$ and then gradually reduced the number of neurons until the performance deteriorated. The process of deleting units, or connections, is usually called "pruning" (Reed, 1993).

We also considered the question of how much training was sufficient. An overtrained NN tends to perform well with the training data set only. We avoided this problem by training the NN until its performance was about the same for both the train- 
ing and the test data set. This technique is known as "early stopping" (Nelson \& Illingworth, 1991).

Dimensioning and training a NN is a trial-anderror process and can be time consuming. However, once a NN is trained, its parameters are fixed. It would not be difficult to implement the NNs in such a way that they can be used in real time, although we did not do so in our project. After each NN was trained, we analyzed its performance based on the results obtained with the testing data set. We used the maximum output as the criteria to determine which output is being activated, that is, all the outputs were compared and the one with the highest value was the one that identified the terrain.

The NN performance was computed using two parameters: Success rate, $S_{R}$, and false alarm rate, $F_{R}$. The success rate performance parameter indicates how often the NN correctly identified each terrain. $S_{R}$ was computed as the ratio between the successfully classified samples, $S_{C S}$, and the total number of samples of the specific terrain being tested, $T_{S}$. It was expressed in terms of a percentage as follows:

$$
S_{R}=100 \frac{S_{C S}}{T_{S T}} .
$$

The false alarm performance parameter indicates how often the NN misclassified a terrain, that is, how often the input sample corresponding to one kind of terrain was classified by the NN as a different type of terrain. $F_{R}$ was computed as the ratio between the number of unsuccessfully classified samples, $U_{C S}$, and the total number of all other samples not corresponding to the type of terrain being tested $T_{S N T}$. The final result was expressed as a percentage using

$$
F_{R}=100 \frac{U_{C S}}{T_{S N T}} .
$$

Desirable performance is characterized by a high $S_{R}$ score and a low $F_{R}$ score. The sum of $S_{R}$ and $F_{R}$ does not have to add up to $100 \%$. In fact, both parameters are independent since one is measured based on samples that correspond to a specific type of terrain (1), while the other is measured based on the samples that do not correspond to the type of terrain to being tested (2).

\subsection{Terrain Classification: Experimental Results}

For the experiments discussed in this section, the P2AT was programmed to travel along a $4 \times 4 \mathrm{~m}$ square-shaped path at a speed of $30 \mathrm{~cm} / \mathrm{s}$. As mentioned before, two experiments on different locations were performed for each terrain; one for training the $\mathrm{NN}$, and the other one for testing and reporting the results. In the remainder of this section, we discuss briefly the peculiarities of each sensor class (Inertial, Motor, Range, Encoder, and Microphone) and provide plots that illustrate some of the results. Due to space limitations, we include plots for only a few of the sensor modalities, specifically, the bestperforming one in each sensor class. Also included with each illustration is a table that summarizes the performance of the respective sensor modality and its associated NN in numeric form. Section 3.3 gives tabular and graphical summaries of the performance of all the sensor modalities and their associated NNs.

\subsubsection{Inertial Sensors: Gyros and Accelerometers}

We created and trained one $\mathrm{NN}$ each for each one of the three onboard gyros $\left(\omega_{x}, \omega_{y}\right.$, and $\left.\omega_{z}\right)$ and for each one of the three onboard accelerometers $\left(a_{x}, a_{y}\right.$, and $\left.a_{z}\right)$. In each case, we used the DFT of the sensor signal as the input for the NN. Figure 6 shows the periodogram for the $X$ axis gyro, $\omega_{x}$, for all five terrains, and Figure 7 shows the resulting NN output. The somewhat confusing-looking Figure 6(b) holds a large amount of information: Each horizontal "row" of plotted data corresponds to one of the five outputs of the NN. For example, the first row represents the "gravel" output, that is, the output of the NN that should ideally be " 1.0 " when the robot traveled over gravel and " 0 " on all other terrains. Each row is divided into five sections, and each section is 170 samples long. Each group of 170 samples was sampled on a different terrain, as follows: First group of 170 samples: Gravel; second group of 170 samples: Grass; third group of 170 samples: Sand; fourth group of 170 samples: Pavement; and fifth group of 170 samples: Dirt.

Table I shows the success rate and the false alarm rate for each terrain in tabular form. The periodogram of all inertial sensors shows the same physical effect: The vibration of the robot seen by the different sensors along their corresponding sensitive axes. Overall, the $X$-axis gyro data produced the best 

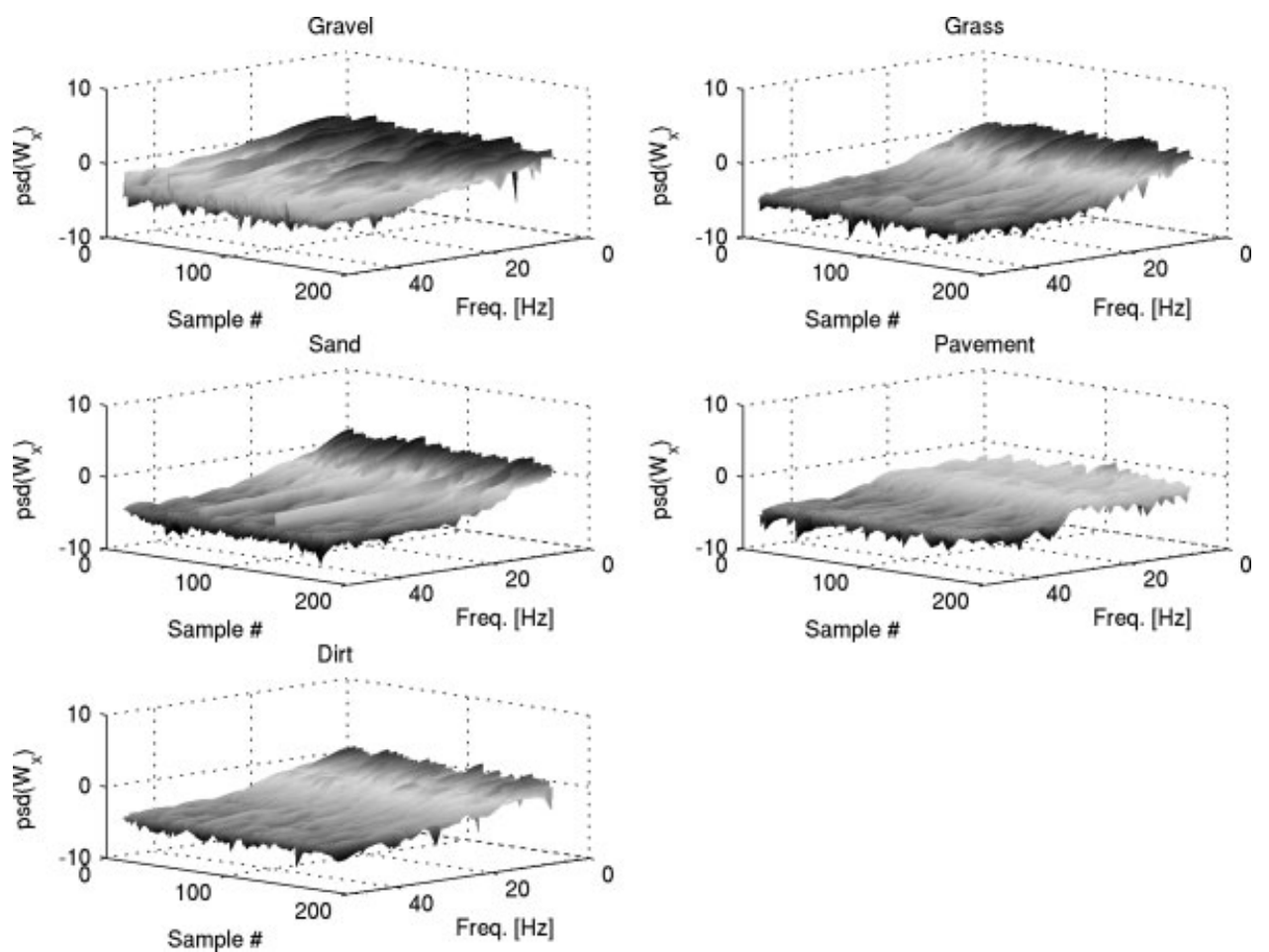

Figure 6. Periodogram of the output of the $x$-axis gyro on the five different terrains.

performance among the inertial sensors. In all cases, the NNs associated with the inertial sensors were quite successful at classifying gravel and pavement. However, these sensors and their associated NNs were less successful on dirt and often failed to distinguish between sand and grass.

\subsubsection{Motor Sensors}

There are four motors on the P2AT; one pair drives the two left-hand wheels, and the other drives the two right-hand wheels. Since the motors of a pair are mechanically linked, we treat each motor pair as a single motor. For terrain classification, two motorrelated sensor modalities are of interest: Current sensors $(I)$ and voltage sensors $(V)$. For each sensor modality, two NNs were used; one to classify terrains using the signal in the frequency domain, and the other one for classification using time domain signals.

Since motor currents vary at much slower frequencies than other observed physical properties of the moving robot (e.g., vibration and dc level), we downsampled the time domain signal of the current measurements by a factor of 5 in order to provide to the NN sufficient significant data to perform the classification. When using the current data in the time domain, the NN performed well for pavement, dirt, and sand, as illustrated in Figures 8 and 9. The good performance with sand could be especially useful if this sensor and its NN were used in combination with inertial sensors, which performed poorly on sand.

For the current sensor data in the frequency domain, the result was a profile similar to that observed with the inertial sensors, but with worse classification results. Also, while previewing the motor sensor data, we found that both the voltage and the current sensors have a low-frequency component that is likely introduced by the controller. For the frequency domain analysis, we computed the difference of the left and right motor currents and voltages, rather than their average. We did so in order to eliminate the effect of the controller-induced lowfrequency component without losing low-frequency terrain information. Since the controller affects both 

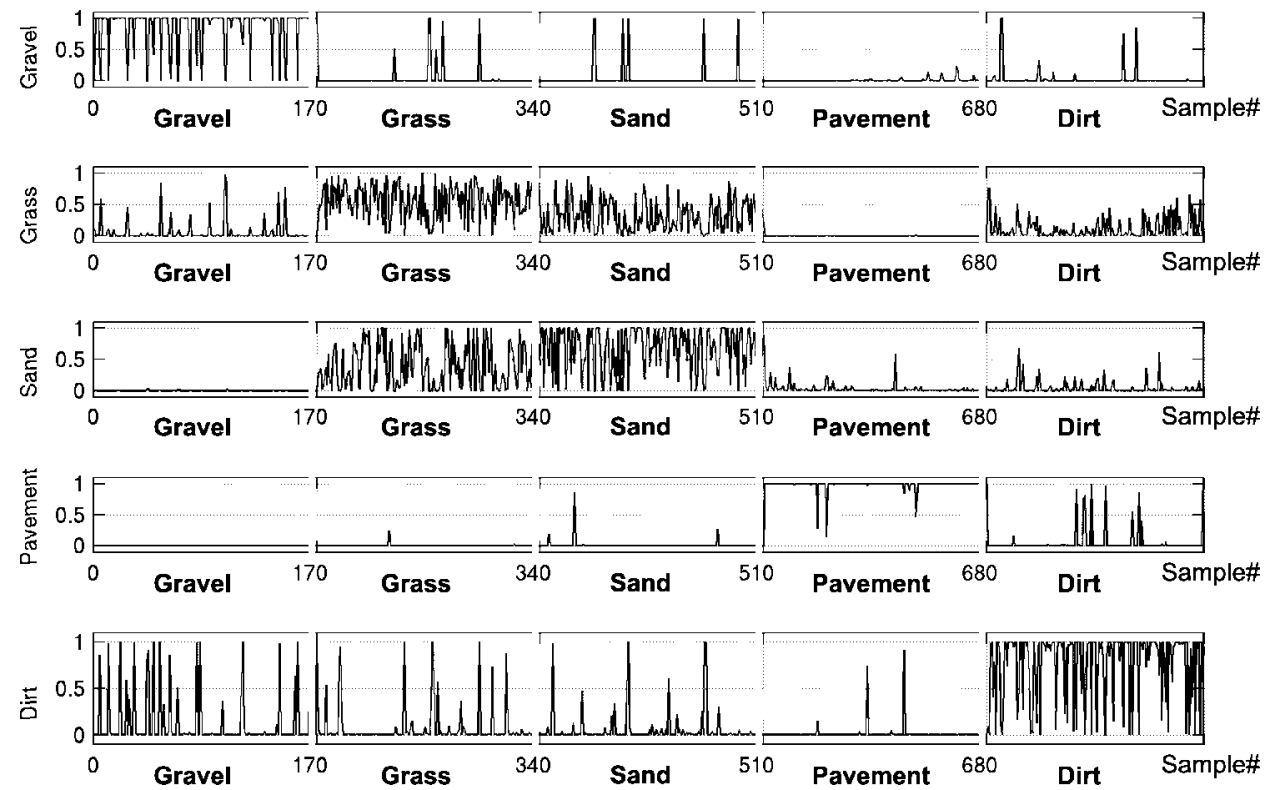

Figure 7. NN output for the $x$-axis gyro after classifying a sequence of DFT sensor data from different terrains. The graph shows a matrix of $(5 \times 170) \times 5$ samples that were fed into the NN. In this matrix, an ideal result would be one in which outputs are 1 on the diagonal and 0 everywhere else. While some of the terrains are very distinguishable, grass is clearly indistinguishable from sand with this sensor modality.

the left and right motors at the same time and to the same degree, this measure completely eliminated the controller-induced low-frequency oscillations. Yet, the variations of the two signals that were due to the interaction between the ground and the wheels were preserved. This difference was then fed into the NN.

Analysis of the motor voltage data, which was also downsampled by a factor of 5 , produced mixed results. The frequency domain data of the voltage sensor and its associated $\mathrm{NN}$ were not very effective

Table I. NN performance of the $X$-axis gyro.

\begin{tabular}{lcc}
\hline & $\begin{array}{c}\text { Success rate } \\
(\%)\end{array}$ & $\begin{array}{c}\text { False alarm } \\
\text { rate }(\%)\end{array}$ \\
\hline Gravel & 90.0 & 2.9 \\
Grass & 71.2 & 7.9 \\
Sand & 70.0 & 6.2 \\
Pavement & 98.8 & 0.9 \\
Dirt & 83.5 & 3.7 \\
Average & 82.7 & 4.3 \\
\hline
\end{tabular}

in classifying terrains. As in the case of the motor current data, the time domain voltage data were more useful, but slightly less so than the motor current data.

\subsubsection{Range Sensors}

We used an infrared (abbreviated "IR") and an ultrasonic range sensor (abbreviated "Son") attached to the robot and pointing downward to the ground (as was shown in Figure 3). In both cases, we classified terrains based on the periodogram of the signal (in the frequency domain) and based on the range signal itself (in the time domain). Both sensors performed marginally better with the frequency domain data, but the overall performance of the NN with these sensors was not as good as that obtained with inertial sensors. Nonetheless, as in the case of the motor voltage sensors, the range sensors and their NNs could be used in a complementary way with the inertial sensors. Figures 10 and 11 show the results for the infrared sensor and the NN classification based on the periodogram of the data. 

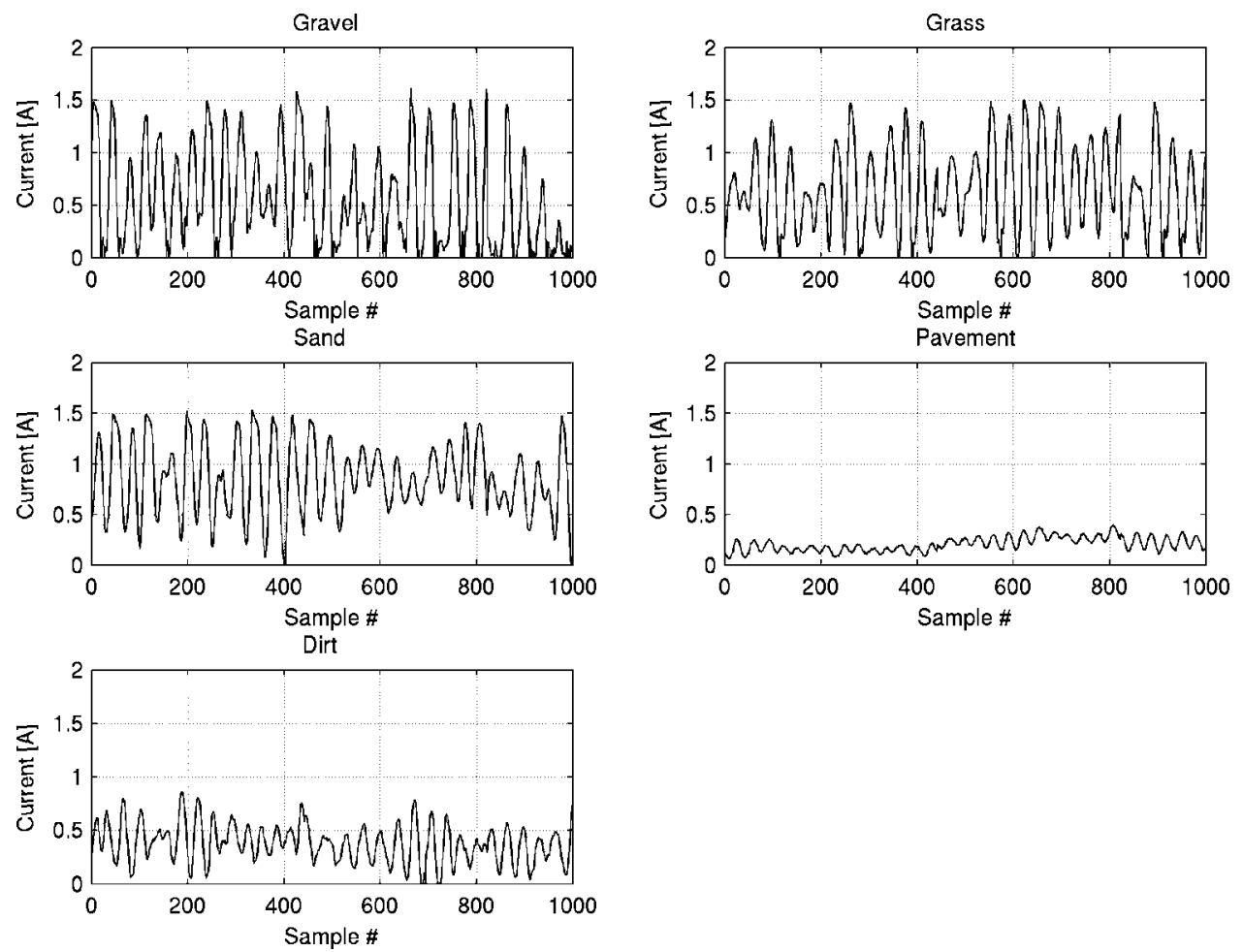

Figure 8. Motor current downsampled raw (time domain) signals for different terrains.

\subsubsection{Microphone}

These signals (abbreviated "Mic") were collected using a downward facing microphone mounted in front of the robot, and sampled using the data acquisition card at $200 \mathrm{~Hz}$. As in the previous cases, we only used the 0 to $50 \mathrm{~Hz}$ components of the DFT transform, after we found that using the whole spectrum did not improve the performance of the NN. Although the overall performance of this sensor modality was not very good, it showed a significantly high success rate for classifying grass.

\subsubsection{Encoders}

We made unconventional use of the left and right wheel encoders (abbreviated "Enc") for terrain classification. At steady state and on smooth terrain, such as pavement, the left wheel encoder produced the same number of tics per sampling interval as the right one did, with minimal variations. On more rugged terrain, however, there were disturbances affecting the wheels and thus the control loops for the left and right motor. Since these disturbances were not the same for both sides, the number of encoder ticks differed more dramatically than on flat terrain. While Table II shows that this approach worked in principle, it did not perform exceptionally well on any terrain.

\subsection{Summary of Experimental Results}

As explained in Section 3.1, we quantified the accuracy of terrain classification with two parameters: success rate, $S_{R}$, and false alarm rate, $F_{R}$. In this section, we define an additional parameter: The classification effectiveness, $C_{E}$. This parameter defines the overall performance of the NN for classifying a specific type of terrain. It is computed as the difference between the classification success rate and false alarm rate:

$$
C_{E}=S_{R}-F_{R} .
$$

As we noted in Section 3.2, the sum of $S_{R}$ and $F_{R}$ 

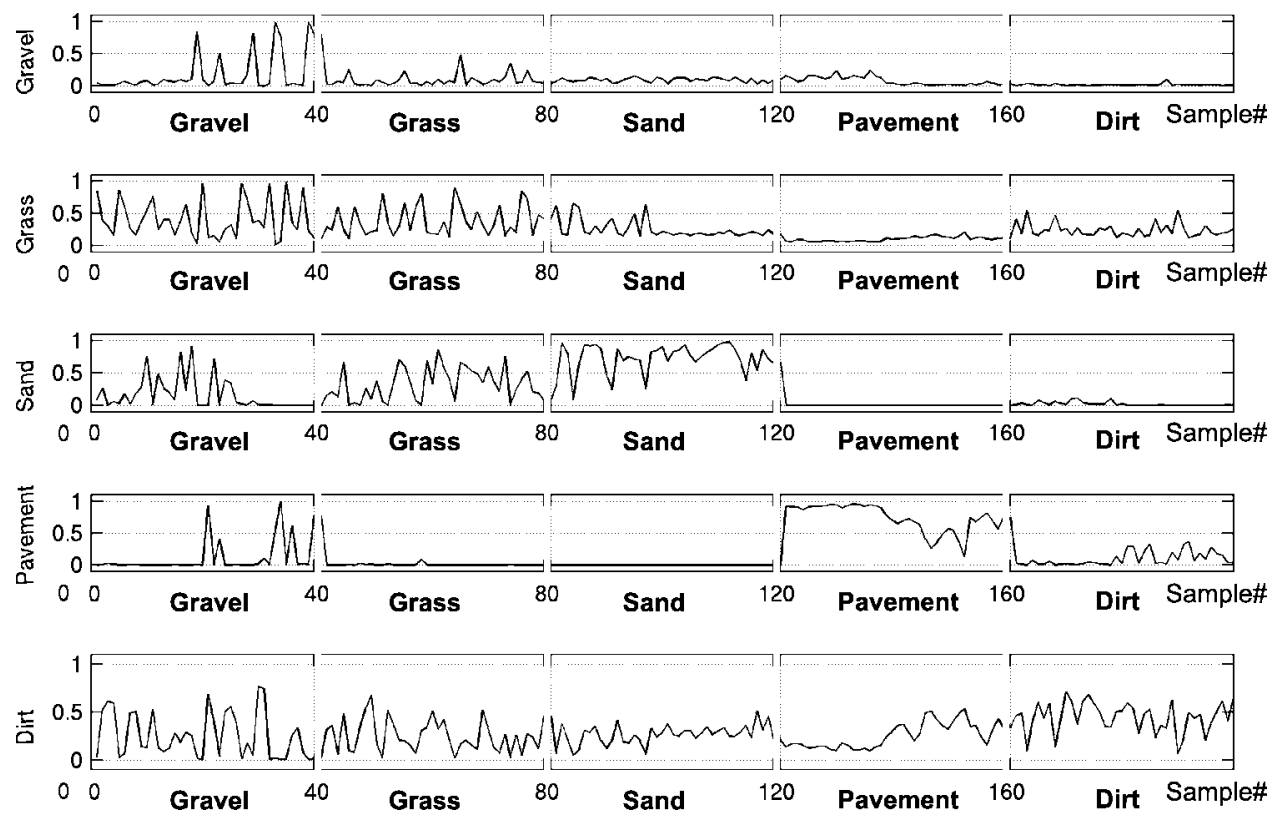

Figure 9. Motor current NN output after classifying time domain sensor data from different terrains (40 inputs per terrain).

does not have to add up to $100 \%$. For example, a poorly trained NN could show $S_{R}=100 \%$ and $F_{R}$ $=100 \%$, which has an effectiveness of $C_{E}=0 \%$.

The $C_{E}$ values for each sensor modality and for each terrain in this study are summarized in Figure 12 and Table II. Figure 13 shows the best and the overall average classification performance for each terrain.

The results of Figure 12 and Table II provide a good idea of which sensor modalities are effective for each terrain. The most useful sensor modality is the gyro, which produced the best performance for three terrains: Gravel, pavement, and dirt. Given that three-axis gyros are commonly found on offroad mobile robots, their data are readily available for terrain classification at no extra hardware cost. Similarly, monitoring the motor currents in the time domain provides the best classification performance for sand; also at no extra hardware cost. Lastly, our study found that the microphone provided the best classification for grass, making that sensor a worthwhile low-cost addition to the sensor suite on mobile robots.

In addition to the performance of individual sensor modalities, we believe that from the results in Table II one can predict which sensor combination might provide the best classification performance. However, in this study, we did not attempt to combine multiple sensor modalities.

\section{TERRAIN TRAFFICABILITY CHARACTERIZATION}

In many applications, knowing on which type of terrain a robot is moving is not sufficient since the same terrain can affect the robot quite differently under different conditions. For example, the driving characteristics will be different on pavement depending on whether it is dry or wet. However, the classification system presented in Section 3 will be able only to determine that the robot is moving on pavement. On the other hand and from the point of view of trafficability, two different terrains can be considered the same if some parameters of interest are identical.

We now propose a method that relates motor currents with rates of turn, through what we call "motor currents versus rate of turn (MCR) curves. Our hypothesis is that key characteristics of the terrain can be identified from MCR curves because there is a strong correlation between motor currents, rates of turn, and 

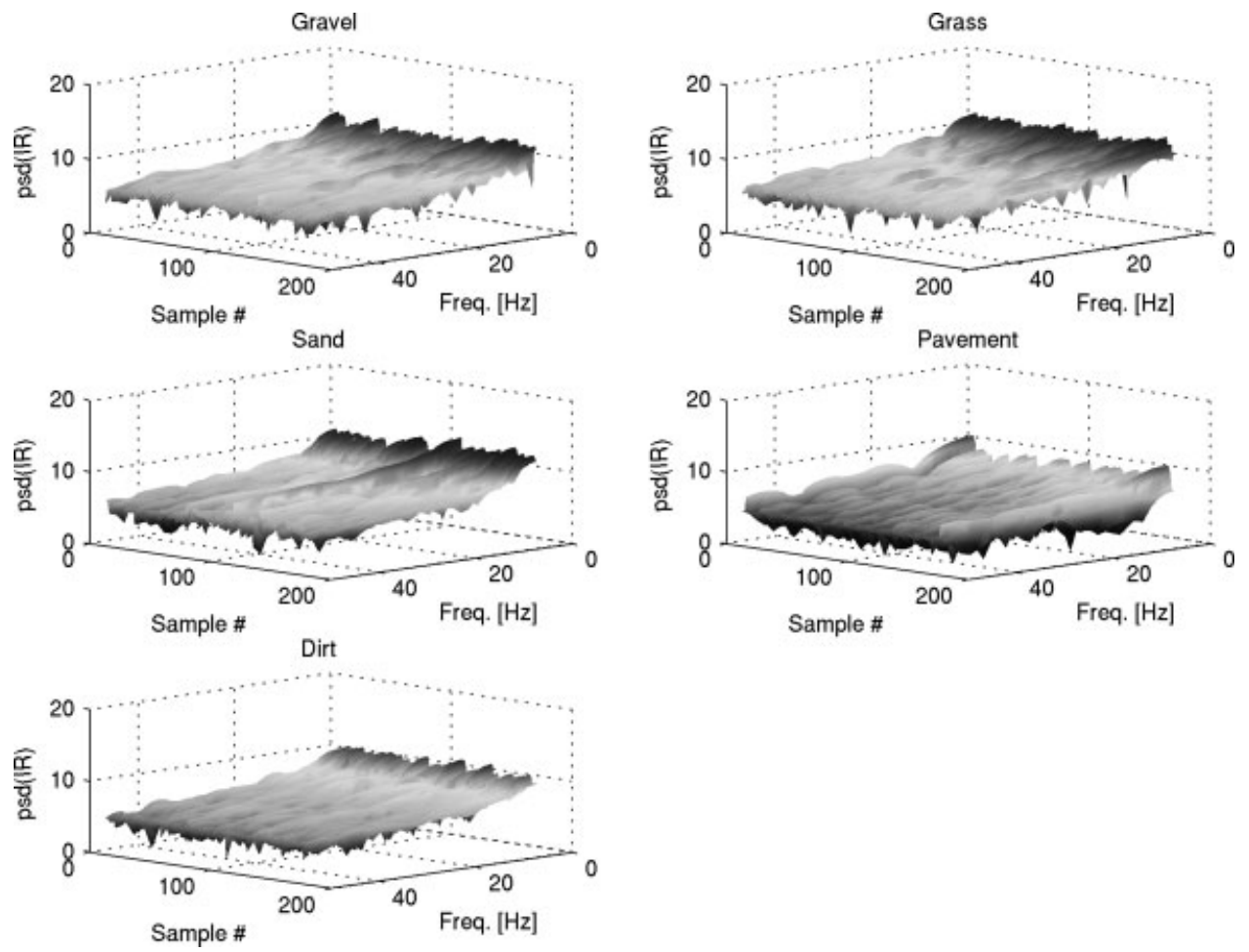

Figure 10. Periodogram of the output of the infrared sensor on the five terrains.

soil parameters. However, before we introduce the MCR method, we present a brief theoretical analysis.

\subsection{Theoretical Analysis}

In the following analysis, we assume that the tires of the robot behave as a rigid rim. This assumption is acceptable for tires with sufficient inflation pressure and stiffness of the carcass (Wong, 2001). For a wheel moving straight on horizontal ground, the average tangential stress, $\tau$, developed in the contact patch with the ground can be estimated using Janosi \& Hanamoto (1961) (see Figure 14):

$$
\tau=(c+\sigma \tan \varphi)\left(1-e^{-j / K}\right),
$$

where $c$ is the cohesion of the soil, $\varphi$ is the internal friction angle of the soil, $\sigma$ is the normal or radial stress, $j$ is the shear displacement, and $K$ is the shear deformation modulus.

The normal or radial stress, $\sigma$, the wheel sink- age, $z$, and the wheel width, $b$, are related according to the following equation (Bekker, 1956):

$$
\sigma(z)=\left(k_{\mathrm{c}}+k_{\varphi} b\right)\left(\frac{z}{b}\right)^{n}
$$

where $k_{c}$ is the cohesive modulus of terrain deformation, $k_{\varphi}$ is the frictional modulus of terrain deformation, and $n$ is the exponent of terrain deformation.

The maximum normal stress occurs at point $\theta_{M}$ and can be computed using (Wong \& Reece, 1967)

$$
\theta_{M}=\left(c_{1}+c_{1} i\right) \theta_{1}
$$

where $\theta_{1}$ is the angle between vertical and leading edge of wheel contact patch, and $i$ is the wheel slippage.

The normal pressure can be split into two regions. The front region $\left(\sigma_{1}\right)$, located between the location of the maximum pressure $\theta_{M}$ and the location of $\theta_{1}$. The rear region $\left(\sigma_{2}\right)$, located between the loca- 

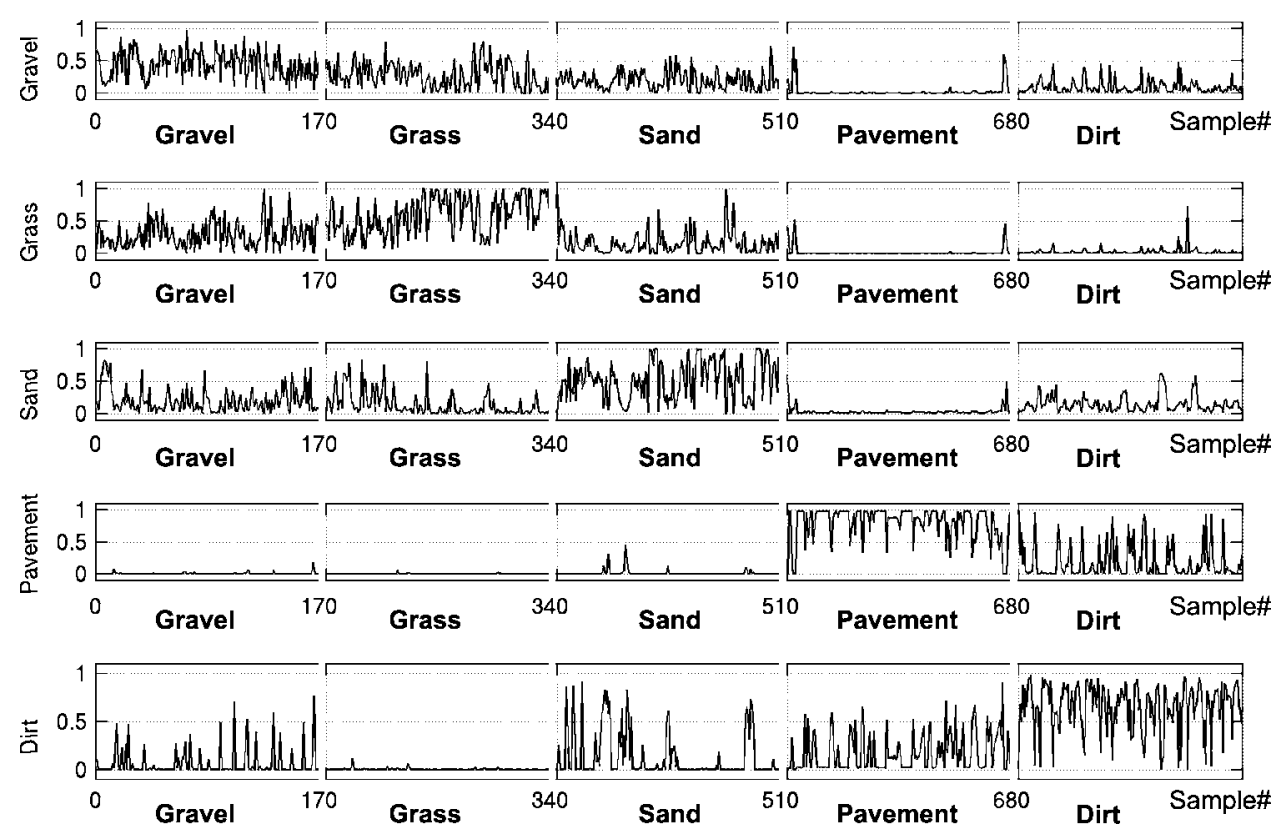

Figure 11. NN output for the infrared sensor after classifying a sequence of DFT sensor data from different terrains. 170 samples per terrain were fed into the $\mathrm{NN}$ and are shown consecutively in each plot.

tions of $\theta_{M}$ and $\theta_{2}$. The angle $\theta_{2}$ is measured between the vertical and trailing edge of the wheel. It is normally small $\left(\theta_{2} \approx 0\right)$ and can be neglected. The normal pressure for the front and rear regions can be computed as a function of the angle $\theta$, as follows (Wong \& Reece, 1967):

$$
\begin{gathered}
\sigma_{1}(\theta)=\left(k_{\mathrm{c}}+k_{\varphi} b\right)\left(\frac{r}{b}\right)^{n}\left(\cos \theta-\cos \theta_{1}\right)^{n}, \\
\sigma_{2}(\theta)=\left(k_{\mathrm{c}}+k_{\varphi} b\right)\left(\frac{r}{b}\right)^{n}\left[\cos \left(\theta_{1}-\frac{\theta}{\theta_{M}}\left(\theta_{1}-\theta_{M}\right)\right)\right. \\
\left.-\cos \theta_{1}\right]^{n} .
\end{gathered}
$$

Shear displacement $j$ is related to wheel slippage $i$ and to angle $\theta$ according to

$$
j(\theta)=r\left[\theta_{1}-\theta-(1-i)\left(\sin \theta_{1}-\sin \theta\right)\right] .
$$

Combining Eqs. (3) and (8), the shear stress around the rim can be calculated as

$$
\tau(\theta)=(\mathrm{c}+\sigma(\theta) \tan \varphi)\left(1-e^{-r / K\left[\theta_{1}-\theta-(1-i)\left(\sin \theta_{1}-\sin \theta\right)\right]}\right) .
$$

The normal stress $\sigma(\theta)$ can be resolved for the front and rear region using Eqs. (6) and (7), respectively. The torque $T$, with which the soil resists the rotation of the wheel, can be computed as the integral of the shear stress over the contact patch with respect to $\theta$ :

$$
T=r^{2} b \int_{\theta_{2}}^{\theta_{1}} \tau(\theta) d \theta
$$

where $r$ is the wheel radius, and $b$ is the wheel width.

Assuming that $\theta_{2}=0$, torque can be obtained from

$$
T=r^{2} b\left\{\int_{\theta_{M}}^{\theta_{1}} \tau_{1}(\theta) d \theta+\int_{0}^{\theta_{M}} \tau_{2}(\theta) d \theta\right\},
$$

Journal of Field Robotics DOI 10.1002/rob 
Table II. NN terrain classification effectiveness for different terrains and sensors. The notation " $(f)$ " indicates that the input variable was used in the frequency domain, while " $(t)$ " indicates that the input variable was used in the time domain. Underlined numbers: best performance; bold numbers: second best performance.

\begin{tabular}{|c|c|c|c|c|c|c|}
\hline & Gravel & Grass & Sand & Pavement & Dirt & Average \\
\hline$\omega_{x}(f)$ & 87.1 & 63.3 & 63.8 & 97.9 & 79.8 & 78.4 \\
\hline$\omega_{y}(f)$ & 91.9 & 46.3 & 47.5 & 91.5 & 77.3 & 70.9 \\
\hline$\omega_{z}(f)$ & 86.1 & 33.7 & 49.3 & 91.4 & 46.0 & 61.4 \\
\hline$a_{x}(f)$ & 84.6 & 52.5 & 41.9 & 78.6 & 44.0 & 60.3 \\
\hline$a_{y}(f)$ & 83.6 & 41.6 & 48.6 & 90.0 & 60.6 & 64.3 \\
\hline$a_{z}(f)$ & 77.7 & 35.9 & 43.0 & 86.9 & 59.0 & 60.6 \\
\hline$I(f)$ & 65.7 & 12.9 & 36.2 & 91.9 & 35.9 & 48.5 \\
\hline$I(t)$ & 12.5 & 30.6 & 81.2 & 83.1 & 76.9 & 56.9 \\
\hline$V(f)$ & 67.8 & 8 & 51.9 & 76.3 & 37.9 & 48.4 \\
\hline$V(t)$ & 1.2 & 35.0 & 78.7 & 80.6 & 70.0 & 53.2 \\
\hline $\operatorname{IR}(f)$ & 41.9 & 64.3 & 50.2 & 91.4 & 69.9 & 63.5 \\
\hline$I R(t)$ & 37.5 & 64.4 & 24.4 & 83.1 & 28.2 & 48.1 \\
\hline $\operatorname{Son}(f)$ & 33.2 & 39.0 & 57.4 & 94.3 & 57.8 & 56.9 \\
\hline $\operatorname{Son}(t)$ & 35.1 & 61.7 & 20.7 & 89.5 & 52.2 & 51.9 \\
\hline $\operatorname{Mic}(f)$ & 76.1 & 72.4 & 37.5 & 87.4 & 28.2 & 60.3 \\
\hline $\operatorname{Enc}(f)$ & 64 & 25 & 64.5 & 91.5 & 57.5 & 60.5 \\
\hline
\end{tabular}

$$
\begin{aligned}
T= & r^{2} b\left\{\int _ { \theta _ { M } } ^ { \theta _ { 1 } } \left[c+\left(k_{\mathrm{c}}+k_{\varphi} b\right)\left(\frac{r}{b}\right)^{n}(\cos \theta\right.\right. \\
& \left.\left.-\cos \theta_{1}\right)^{n} \tan \phi\right]\left(1-e^{-r / K\left[\theta_{1}-\theta-(1-i)\left(\sin \theta_{1}-\sin \theta\right)\right]}\right) d \theta \\
& +\int_{0}^{\theta_{M}}\left[( k _ { \mathrm { c } } + k _ { \varphi } b ) ( \frac { r } { b } ) ^ { n } \left(\cos \left(\theta_{1}-\frac{\theta}{\theta_{M}}\left(\theta_{1}-\theta_{M}\right)\right)\right.\right. \\
& \left.\left.-\cos \theta_{1}\right)^{n} \tan \phi\right]\left(1-e^{-r / K\left[\theta_{1}-\theta-(1-i)\left(\sin \theta_{1}-\sin \theta\right)\right]}\right) d \theta .
\end{aligned}
$$

In order to solve Eq. (10), $\theta_{1}$ must be determined. For this purpose, we use the following equation (Wong \& Reece, 1967):

$$
W=r b\left(\int_{\theta_{2}}^{\theta_{1}} \sigma(\theta) \cos \theta d \theta+\int_{\theta_{2}}^{\theta_{1}} \tau(\theta) \sin \theta d \theta\right) .
$$

Equation (12) is too complex and cannot be solved analytically. However, provided that $W$ is known, the right side of Eq. (12) can be computed numerically for different values $\theta_{1}$ until a value is found that solves the equation. For a detailed explanation of this method see Wong \& Reece (1967). Once the torque has been determined, the motor current $I$, which is known to be roughly proportional to torques applied to the wheels, can be determined according to

$$
T=k_{I} I .
$$

The constant $k_{I}$ is the torque scale factor. By combining Eqs. (11) and (13), motor currents and slippage can be related, provided that all the other parameters are known. Using soil parameters for sand (Wong, 2001) (see Table III) and the parameters of P2AT, we plotted Figure 15, which shows the relationship between current $I$ and slip $i$. This graph was 


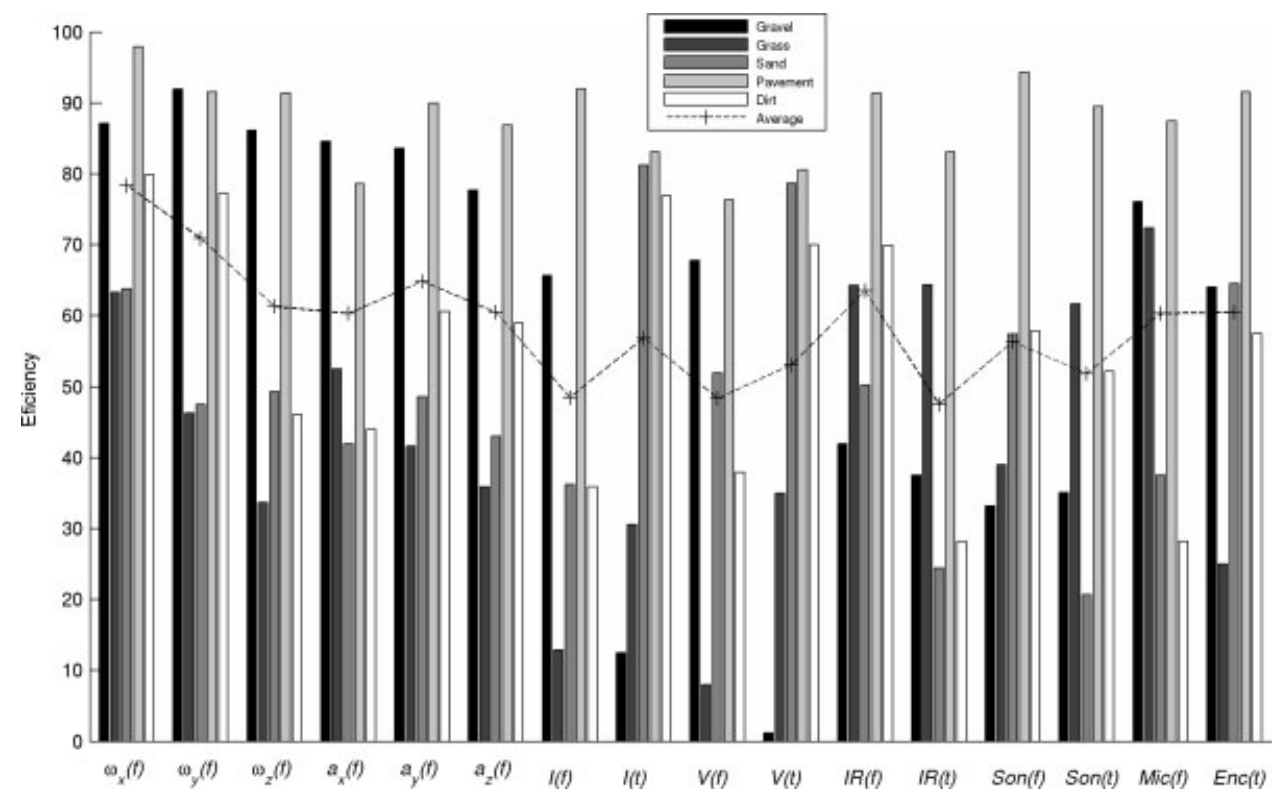

Figure 12. Terrain classification effectiveness for different sensors and terrains (higher values are better).

created by simulating different amounts of slippage and solving Eqs. (5)-(13). The integrals were evaluated numerically using the Simpson method.

As is evident in Figure 15, the shape of the slippage versus motor current relationship depends on the robot and terrain parameters. Although it is not

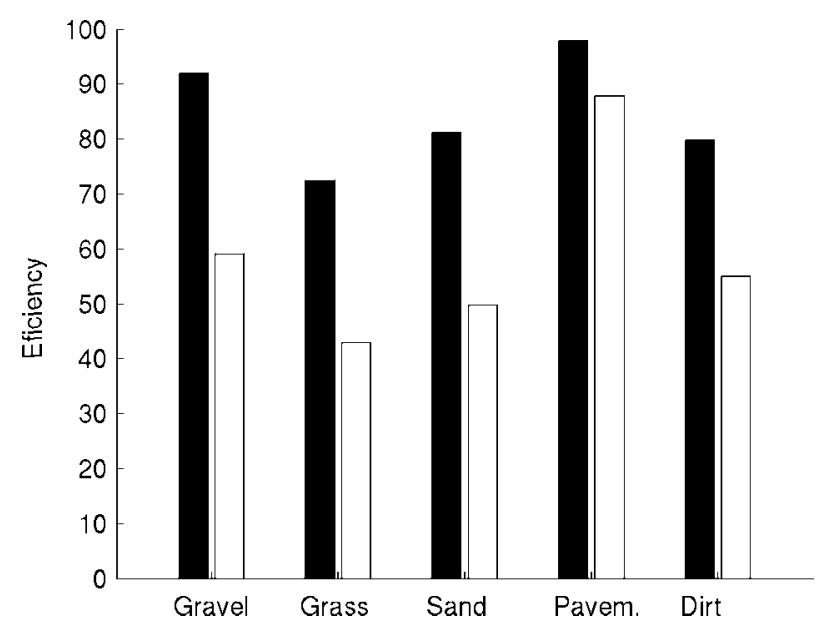

Figure 13. Classification effectiveness for different terrains. Black: best performance; white: average performance. possible to determine the soil parameters form these relationships, it shows important information about the soil conditions as explained in Ojeda, Cruz, Reina \& Borenstein (2005). In Ojeda, Cruz, Reina \&

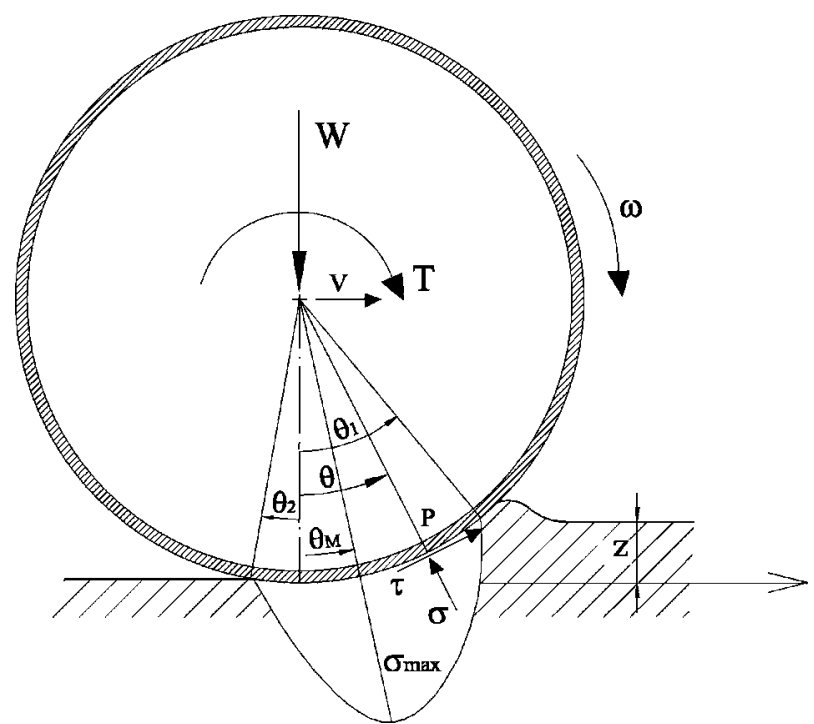

Figure 14. Wheel-soil interaction model (adapted from Bekker, 1969). 
Table III. Sand parameters used for simulations.

\begin{tabular}{lc}
\hline Parameter & Value \\
\hline$\varphi(\mathrm{deg})$ & 28.00 \\
$c(\mathrm{kPa})$ & 1.04 \\
$K(\mathrm{~m})$ & 0.025 \\
$k_{c}\left(\mathrm{kN} / \mathrm{m}^{\mathrm{n}+1}\right)$ & 0.99 \\
$k_{\varphi}\left(\mathrm{kN} / \mathrm{m}^{\mathrm{n}+2}\right)$ & 1528.43 \\
$n$ & 1.10 \\
$c_{1}$ & 0.18 \\
$c_{2}$ & 0.32 \\
\hline
\end{tabular}

Borenstein (2005), some techniques are proposed for automatic creation of the current versus slip curves, but they are either limited for one specific terrain (sand) or not immediately applicable for a skid-steer platform.

An important characteristic about skidsteering is that slippage is induced when the robot turns; however, in this case the amount of slippage in the outer and inner tires is different. The following relationship applies (Wong, 2001):

$$
\omega=\frac{r \omega_{o}\left(1-i_{o}\right)-r \omega_{i}\left(1-i_{i}\right)}{B}
$$

where $\omega$ is the rate of turn of the robot, $I_{o, I}$ is the outer and inner slippage, $\omega_{0, I}$ is the outer and inner

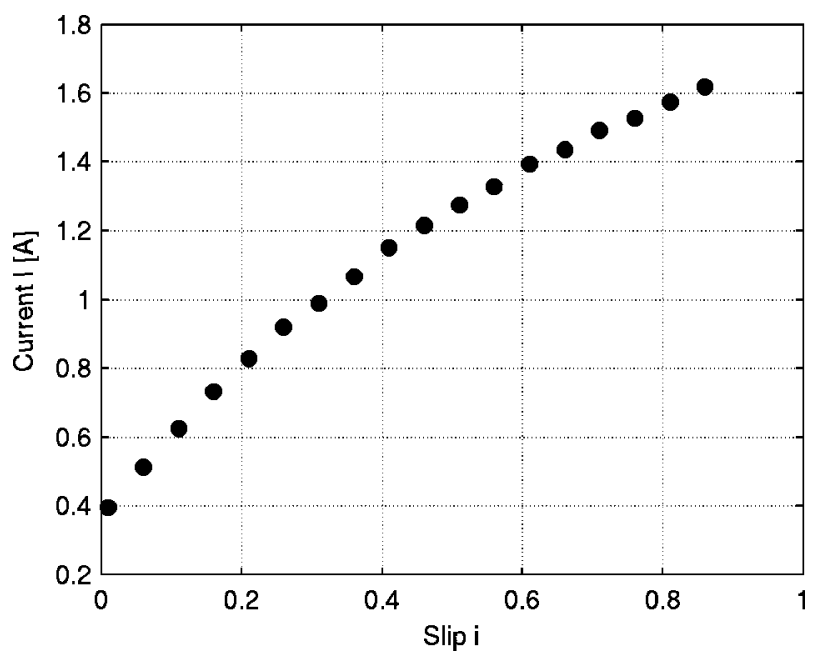

Figure 15. Theoretical wheel slippage vs motor currents relationship.

Journal of Field Robotics DOI 10.1002/rob angular rate of the wheel, and $B$ is the track of the vehicle.

Therefore, by monitoring motor currents and rate of turn, we can determine the MCR curves which can be used to characterize the terrain. The tangential stress for the outside, $\tau_{0}$, and inside wheels, $\tau_{i}$, are also different and can be expressed as follows (Wong \& Chiang, 2001):

$$
\begin{gathered}
\tau_{o}=\left(\mathrm{c}+\sigma_{o} \tan \varphi\right)\left(1-e^{-j_{o} / K}\right), \\
\tau_{i}=\left(\mathrm{c}+\sigma_{i} \tan \varphi\right)\left(1-e^{-j_{i} / K}\right) .
\end{gathered}
$$

The equation for computing the torque when the robot turns is different than the one for straight motion (Wong \& Chiang, 2001):

$$
\begin{aligned}
& T_{o}=r \int_{A} \tau_{o} \sin \delta_{0} d_{A}, \\
& T_{i}=r \int_{A} \tau_{i} \sin \delta_{i} d_{A},
\end{aligned}
$$

where the angles $\delta_{0}$ and $\delta_{i}$ are measured between the sliding velocities and the lateral directions of the wheels. Equations (17) and (18) have been included in this paper for completeness, and we have not attempted to simulate them in this work. A detailed explanation of their use for tracked vehicles can be found in the general theory of skidsteering on firm ground proposed in Wong \& Chiang (2001).

\subsection{Terrain Characterization: Experimental Results}

The following experiments where performed on five different surfaces: Gravel, grass, sand, and pavement. For this purpose, we commanded the P2AT to move at a constant linear speed of $200 \mathrm{~mm} / \mathrm{s}$, while the rate of turn was increased by $2.0^{\circ} / \mathrm{s}$ every $15 \mathrm{~s}$ up to $30^{\circ} / \mathrm{s}$. Therefore, the method, as described, is not particularly suitable for terrain characterization in real time, during an actual robot mission. In Section 4.3 , we present three possible real-time implementations that overcome this problem. Nevertheless, this method is the most accurate and it is advisable to use it at the time of generating reference curves. 

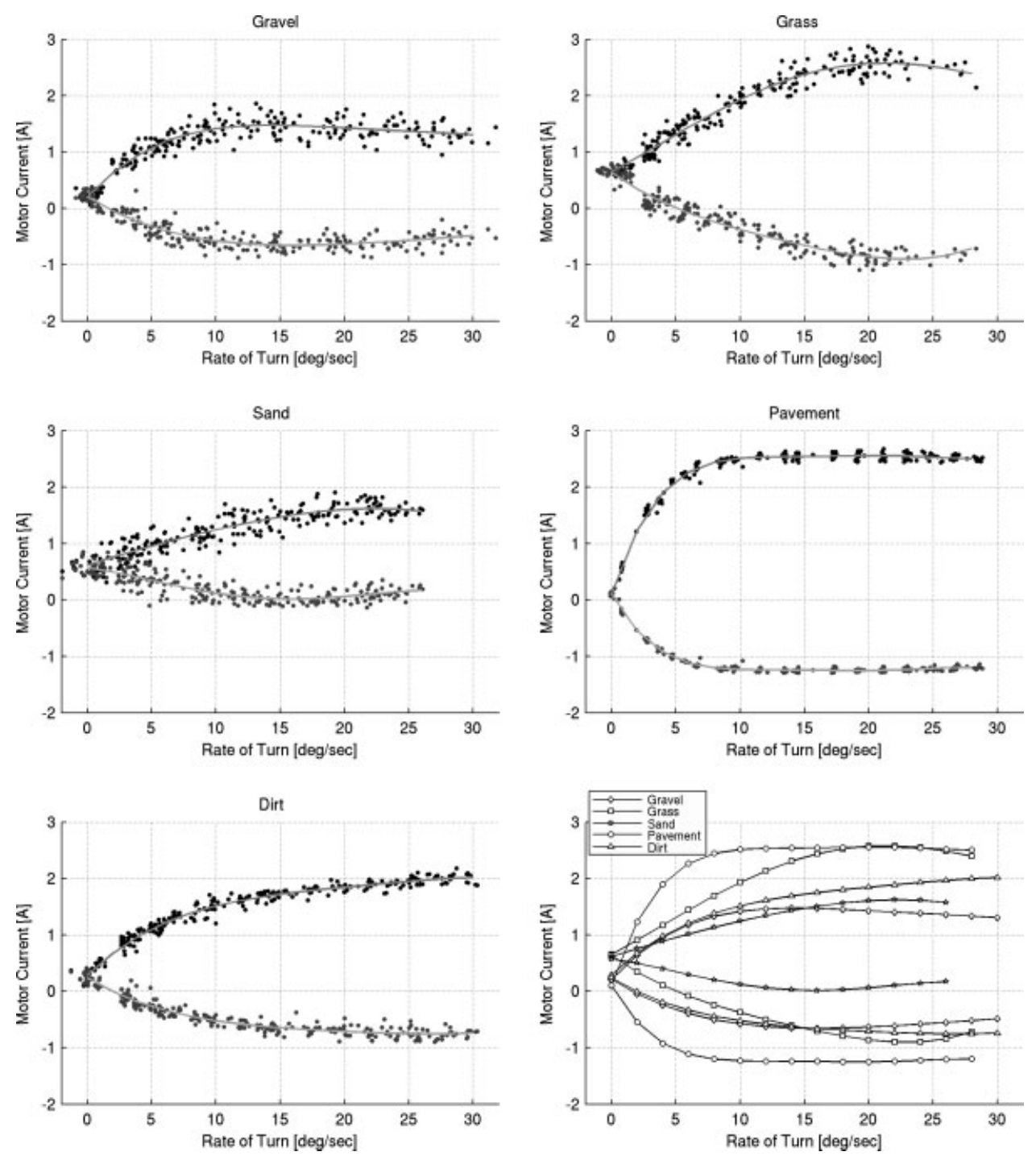

Figure 16. MCR curve characteristic for each one of the tested terrains. Black dots: outer wheel; dark gray dots: inner wheel; and light gray solid line: the polynomial fit. The lower right plot shows the polynomial fit of the MCR curves for all the tested terrains.

The MCR curve establishes the relationship between motor currents and the angular rate of the robot. Since these two parameters are affected by the floor characteristics (i.e., soil parameters), they can be used to determine terrain characteristics. Figure 16 shows the MCR curve characteristics obtained for each one of the test surfaces. In this plot, the $x$ axis represents the angular rate of the robot as measured by the gyroscope and the $y$ axis shows the associated motor current measured on the outer and inner mo- tors. For each MCR curve, we fit a fourth-order polynomial function to the data. The variance of the curves from the polynomial fit is due to the disturbances caused by rugged terrain and wheel slippage. For comparison purposes, Figure 16 also includes the resulting polynomial curves for all the terrains in one single plot.

The MCR curve can be used to predict the power requirements of the robot while traveling on a specific terrain, since it measures the actual motor 
currents and the operating voltage is known. Another use of the MCR curve is for determining driving parameters for safe handling on the specific terrain.

The MCR curve can also be used to determine the coefficient of motion resistance, $f_{r}$. Motion or roll resistance is the force that opposes the torque generated by the drive motor(s). Motion resistance is the combined effect of friction, hysterics, surface condition, tire inflation, wind resistance, etc. (Bekker, 1960).

At low speeds, when the robot is turning at a constant rate, the sum of all the tangential forces is equal to zero; therefore, the following relations apply:

$$
\begin{gathered}
\sum F_{t}=0, \\
F_{o}+F_{i}=R_{t o}+R_{t i},
\end{gathered}
$$

where $F_{o, i}$ is the outer and inner force developed in the wheel contact patch, and $R_{t o, i}$ is the external motion resistances on the outer and inner wheels.

The forces $F_{o}$ and $F_{i}$ can be computed by means of the motor currents using

$$
\begin{aligned}
& F_{o}=T_{o} r=k_{I} I_{o} r, \\
& F_{i}=T_{i} r=k_{I} I_{i} r,
\end{aligned}
$$

where $r$ is the wheel radius. Using $R_{o}$ and $R_{i}$, the coefficient of motion resistance, $f_{r}$, can be computed according to

$$
f_{r}=\frac{\left(R_{t o}+R_{t i}\right)}{W}
$$

where $W$ is the weight supported by each wheel. The coefficient of motion resistance for all five terrains is shown in Figure 17.

The MCR relationship can be affected considerably by many factors, such as moisture content, surface structure, or stratification (formation of layers) of soil. In order to demonstrate this point, we collected data on pavement, before and after rainfall. The resulting MCR curves are clearly different, as is evident from Figure 18. This can be a problem if the

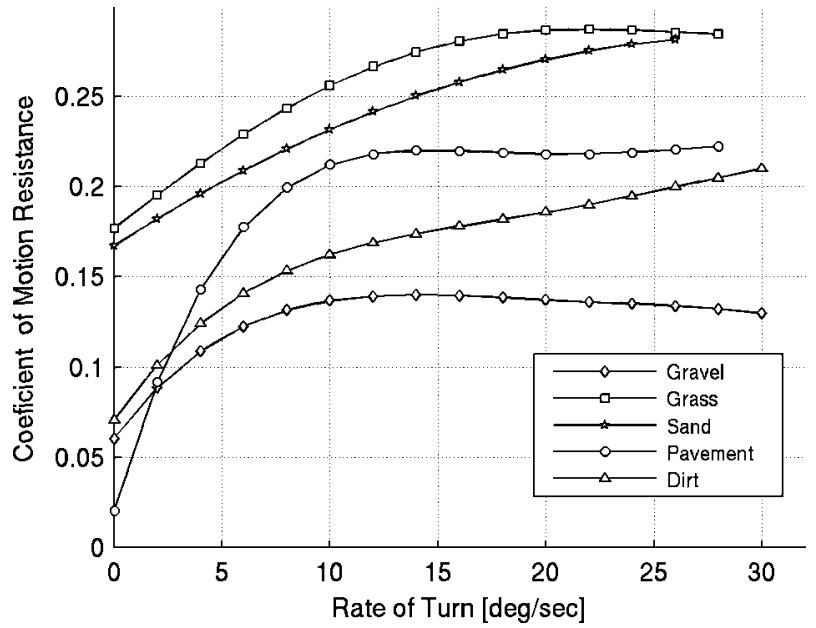

Figure 17. Coefficient of motion resistance for different terrains at different rates of turn. These data were obtained directly from the polynomially fitted curves of Figure 16.

goal is terrain classification. However, from the trafficability point of view, the two surface conditions are indeed different, even though they were measured on the same terrain. The coefficient of motion resistance for this experiment is shown in Figure 19.

There is an additional benefit: By measuring momentary torques applied to the wheels and comparing those with the MCR curve, one can estimate the

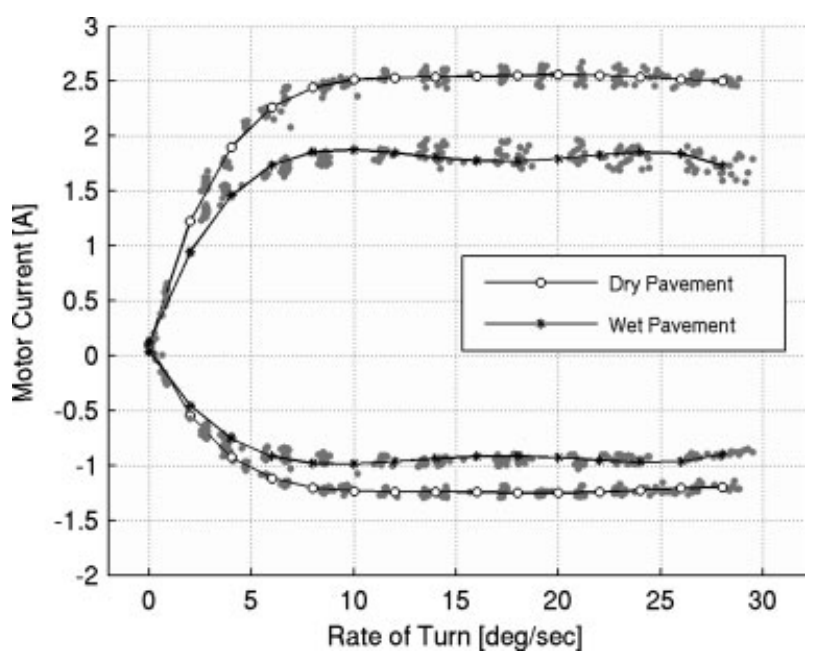

Figure 18. MCR curve characteristic and polynomial fit for wet pavement and dry pavement. 


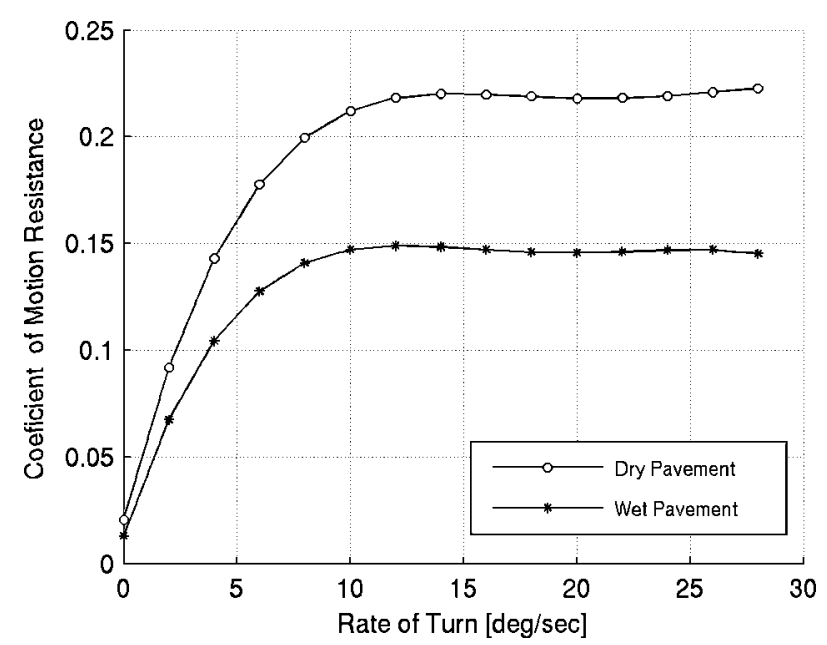

Figure 19. Coefficient of motion resistance for wet pavement and dry pavement. These data were obtained directly from the polynomially fitted curves of Figure 18.

amount of wheel slippage incurred on that terrain. This, in turn, allows detecting and correcting (to some degree) odometry errors. The authors developed such a technique in earlier work for planetary rovers, with consistently good results (Ojeda, Cruz, Reina \& Borenstein, 2005). Also, as is evident in Figure 16, some curves can be distinguished easily, as in the case for pavement. This suggests that it is possible to perform terrain classification using the MCR curves alone as reported by the authors in Ojeda, Borenstein \& Witus (2005).

\subsection{Real-Time Implementation}

A drawback of the above described method is that it requires a significant amount of time and a fairly large ground surface area. In this section, we propose three methods that require less ground area and are suitable for real-time implementation.

The first real-time implementation is a direct extension of the basic approach presented in Section 4.2. The only difference is that the robot stays at each rate of turn for shorter periods of time. A tradeoff exists between the amount of measurement noise and the length of periods, at which each rate of turn is held constant, especially on terrains that produce noisy data, such as grass or gravel. Longer constantrate periods allow some averaging of the motor current and rate of turn data pairs, thereby significantly reducing the effect of noise. With very short constant-rate periods, on the other hand, the MCR curve may not accurately represent the terrain characteristics.

In the second approach, the robot is subject to sinusoidal rates of turn commands. This is done by commanding the robot to move straight at a constant speed, while a sinusoidal changing rate of turn command is overlaid over the straight motion command. This method works well because it allows collecting redundant data through multiple sinusoidal cycles, while still being of short duration, since the rate of turn is varied rapidly and continually. Furthermore, it does not impose large changes in the trajectory. Therefore, it is feasible to apply this method while the robot moves toward a goal. The sinusoidal path perturbations imposed by this method cause only small deviations from the desired straight-line path during a mission.

We also developed and tested a variation of the previous method. With this method, the robot is subject to varying-frequency sinusoidal rates of turn commands. As with the previous method, this method allows the robot to collect redundant data over the course of several sinusoidal-rate cycles. The robot can progress toward the target, without deviating significantly from its course. Varying the frequency of the sinusoidal rate allows determining the frequency response of the robot to changes in rate of turn. The slippage conditions also vary with the frequency. The authors successfully demonstrated how this method can be used for performing terrain classification based on MCR curves (Ojeda, Cruz, Reina \& Borenstein, 2005).

These three real-time variants for creating MCR curves reduce not only the time necessary for collecting the data, but they also reduce the size of the terrain area necessary to collect the data. On the other hand, the robot hardware may impose practical limitations on changes of the rate of turn. For example, in the P2AT that we used in our experiments, the maximum update rate of the internal microcontroller is 10 commands/s, and the robot can only be commanded to increase rates of turn in increments of $1^{\circ} / \mathrm{s}$.

\section{CONCLUSION}

This paper addresses two related topics in mobile robotics: Terrain classification and trafficability charac- 
terization. The presented method for terrain classification is based on the analysis of multiple sensor modalities with NNs. Among all tested sensor modalities, the $X$-axis gyro provides the best signal for NN-based terrain classification purposes. The inertial sensors are good at distinguishing terrains as long as the terrain causes distinct vibrations. For the five tested terrains, the NNs associated with the inertial sensors were capable of classifying gravel and pavement very well. The performance was not so good when classifying dirt, which was often confused with sand and grass.

As noted throughout this paper, we believe that the performance of an NN-based terrain classification system can be improved by combining multiple sensor modalities, although we did not attempt to do that in this study. There are two reasons for this assessment. The first and obvious reason is that some sensor modalities and their NNs performed better than others. The second, less obvious reason is that the combination of multiple sensor modalities in a single NN would allow the designer to train each NN for its "preferred" terrain instead of for all terrains. For example, the $X$-axis gyro sensor $G_{X}(f)$ could be used to classify gravel, pavement, dirt, and "grass $O R$ sand;" (where "OR" is the Boolean OR operator), while the voltage sensor $\operatorname{Volt}(t)$ can be trained to recognize "only sand."

We also introduced the concept of MCR curves, which contain important information about the soil parameters. These MCR curves can be used for different purposes: Predicting power consumption requirements, determining driving parameters for safe handling, and estimating the coefficient of road resistance. In this paper, we presented three possible approaches for real-time implementation of this method, which can be used during an actual mission.

In future work, it might be possible to predict the vehicle's handling and performance on new upcoming terrain, if the system was coupled with an appropriate look-ahead sensing system.

\section{ACKNOWLEDGMENTS}

This research was performed under subcontract to Turing Associates, Inc., of Ann Arbor, MI, as part of SBIR Contract No. DAAE07-02-C-L003 to the U.S. Army RDECOM (TARDEC). Publication of these results does not constitute endorsement by an agency or official of the U.S. Government.

\section{REFERENCES}

Bekker, G. (1956). Theory of land locomotion. Ann Arbor, MI: University of Michigan Press.

Bekker, G. (1960). Off the road locomotion. Ann Arbor, MI: University of Michigan Press.

Bekker, G. (1969). Introduction to terrain-vehicle systems. Ann Arbor, MI: University of Michigan Press.

Belhadj, Z., Saad, A., El Assad, S., Saillard, J., \& Barba, D. (1994, April). Comparative study of some algorithms for terrain classification using SAR images. Paper presented at International Conference on Acoustics, Speech, and Signal Processing, Adelaide, Australia, vol. 5, pp. 165-168.

Brooks, C., Iagnemma, K., \& Dubowsky, S. (2005, April). Vibration-based terrain analysis for mobile robots. Paper presented at IEEE International Conference on Robotics and Automation, Barcelona, Spain, pp. 18-22.

Hornik, M., Stinchcombe, M., \& White, H. (1989). Multilayer feedforward networks are universal approximators. Neural Networks, 2(5), 359-366.

Iagnemma, K., Kang, S., Shibly, H., \& Dubowsky, S. (2004, October). On-line terrain parameter estimation for planetary rovers. IEEE Transactions on Robotics, 20(2), 921-927.

Janosi, Z., \& Hanamoto, B. (1961). Analytical determination of drawbar pull as a function of slip for tracked vehicles in deformable soils. Paper presented at First International Conference on Terrain-Vehicle Systems, Torino, Italy.

Larson, A., Voyles, R., \& Demir, G. (2004, April-May). Terrain classification through weakly structured vehicle/ terrain interaction. Paper presented at IEEE International Conference on Robotics and Automation, New Orleans, LA, Vol. 1, pp. 218-224.

Matijevic, J., Crisp, J., Bickler, D., Banes, R., Cooper, B., Eisen, H., Gensler, J., Haldemann, A., Hartman, F. Jewett, K., Matthies, L., Laubach, S., Mishkin, A., Morrison, J., Nguyen, T., Sirota, A., Stone, H., Stride, S., Sword, L., Tarsala, J., Thompson, A., Wallace, M., Welch, R., Wellman, E., Wilcox, B., Ferguson, D., Jenkins, P., Kolecki, J., Landis, G., \& Wilt, D. (1997). Characterization of the Martian surface deposits by the Mars Pathfinder rover, Sojourner. Science, 278, 17651768.

Nelson, M., \& Illingworth, W. (1991). A practical guide to neural nets. Reading, MA: Addison-Wesley.

Nohse, Y., Hashiguchi, K., Ueno, M., Shikanai, T., Izumi, H., \& Koyama, F. (1991). A measurement of basic mechanical quantities of off-the-road traveling performance. Journal of Terramechanics, 28(4), 359-370.

Ojeda, L., Borenstein, J., \& Witus, G. (2005, March-April). Terrain trafficability characterization with a mobile robot. Paper presented at SPIE Defense and Security Conference, Unmanned Ground Vehicle Technology VII, Orlando, FL.

Ojeda, L., Cruz, D., Reina, G., \& Borenstein, J. (2005). Current-based slippage detection and odometry correction for mobile robots and planetary rovers. IEEE Transactions on Robotics (to be published).

Ojeda, L., Reina, G., \& Borenstein, J. (2004, March). Experi- 
mental results from FLEXnav: An expert rule-based dead-reckoning system for Mars rovers. Paper presented at IEEE Aerospace Conference, Big Sky, MT.

Papoulis, A. (1991). Probability, random variables and stochastic processes. New York: McGraw-Hill.

Reed, R. (1993). Pruning algorithms-a survey. IEEE Transactions on Neural Networks, 4, 740-747.

Sadhukhan, D. (2004). Autonomous ground vehicle terrain classification using internal sensors. M.S. thesis, Florida State University, Tallahassee, FL.

Shmulevich, I., Ronai, D., \& Wolf, D. (1996). A new field single wheel tester. Journal of Terramechanics, 33(3), 133-141.

Talukder, A., Manduchi, R., Castano, R., Owens, K., Matthies, L., Castano, A., \& Hogg, R. (2002, September). Autonomous terrain characterization and modelling for dynamic control of unmanned vehicles. Paper presented at IEEE Conference on Intelligent Robots and Systems (IROS), Lausanne, Switzerland.

Vandapel, N., Huber, D., Kapuria, A., \& Hebert, M. (2004,
April-May). Natural terrain classification using 3D Ladar data. Paper presented at IEEE International Conference on Robotics and Automation, New Orleans, LA, pp. 5117-5122.

Weszka, J., Dyer, C., \& Rosenfeld, A. (1976). A comparative study of texture measures for terrain classification. IEEE Transactions on Systems, Man and Cybernetics, 6, 269-285.

Wong, J. (2001). Theory of ground vehicles. New York: Wiley-Interscience.

Wong, J., \& Chiang, C. (2001). A general theory for skidsteering of tracked vehicles on firm ground. Proc. Institution of Mechanical Engineers, Part D, Journal of Automobile Engineering, 215(3), 343-355.

Wong, J., \& Reece, A. (1967). Prediction of rigid wheel performance based on the analysis of soil-wheel stresses, part I. Journal of Terramechanics, 4(1), 81-98; Prediction of rigid wheel performance based on the analysis of soil-wheel stresses, part II. ibid., 4(2), 7-25. 